\title{
NEWSART: Negative binomial weighted spectral analysis in real time
}

\author{
PHILLIP L. EMERSON \\ Cleveland State University, Cleveland, Ohio
}

\begin{abstract}
In this paper, digital filters are described for concurrent adaptive estimation of the spectral powers at several target frequencies. Least-squares estimation is used, with a tapered weight function that discounts time-series data according to age. Frequency selectivity and reliability of spectral estimates are controlled by different parameters. The spectral windows are closely approximated by Student's $t$ distribution, which is useful in the planning of experiments.
\end{abstract}

It is possible to perform adaptive digital estimation of a few spectral components from a running-time series using ordinary personal computers. Recent increases in central processing unit speed, combined with recursive computing techniques, have extended the estimable frequencies into the lower part of the audible range.

Recursive computing techniques concerning adaptive real-time estimation of the two Fourier coefficients for a single target frequency from a running-time series were first described by Emerson (1985). The adaptiveness was obtained by least-squares estimation with a geometrically decaying weight function that discounted old data according to age. The sum of the squares of the recursively estimated Fourier coefficients provided a running numerical estimate of the spectral power at and near the target frequency. The main application of this technique was for biofeedback training in which a subject was to learn to produce EEG waves in the vicinity of a specified target frequency. With one EEG application, a sampling rate of $40 \mathrm{~Hz}$ was used for estimating the power at a target frequency of $10 \mathrm{~Hz}$.

With the increasing speed of computations on newer model personal computers, it is feasible to extend such filtering to higher frequencies and to obtain simultaneous estimations at several target frequencies. Moreover, the filter characteristics can be controlled better by generalizing the weight function to the negative binomial family within which the geometric series is the simplest member.

With such generalizations, the range of potential applications has grown beyond the domain of EEG feedback with a single target frequency, into other areas in which real-time decisions must be based on timely information about the changing power spectrum of a runningtime series. For example, Sturgis (1983) recorded automobile steering-wheel movements of experienced and inexperienced drivers. Off-line Fourier analysis revealed different spectral patterns for the two classes of drivers. The experienced drivers generally showed lower amplitude but higher frequency steering corrections. It is also

The author's mailing address is: Department of Psychology, Cleveland State University, Cleveland, OH $\mathbf{4 4 1 1 5 .}$ possible to study the spectral patterns obtained under a variety of other driving parameters such as fatigue, sleep deprivation, and drug intoxication. Such research could contribute to the design of inexpensive automated electronic modules for the real-time analysis of steering-wheel movements, and for the detection of spectral patterns that diagnose dangerous conditions.

NEWSART filtering fills a niche between those of realtime analog hardware filtering and off-line spectral analysis using the fast Fourier transform (FFT). The FFT is rarely used in real time with small general-purpose computers because it requires too many computations, and the updated results become available only at intervals equal to the length of the record segment. Hardware analog filters, on the other hand, provide almost instantaneous computations, and their results are available continuously. However, they are relatively expensive and inflexible. NEWSART filters provide a compromise that is advantageous under some circumstances. New outputs are available at the same rate as the discrete, equally spaced input observations, and parameter values are programmable. However, the computations take time, which places limits on the sampling rate and on the number of components to be estimated.

The equipment needed for research with NEWSART filters are: hardware signal preconditioning equipment such as an analog bandpass filter and amplifier, an analog-todigital (A/D) converter with moderately fast conversion time, a programmable real-time clock to trigger the equally spaced conversions, and a general-purpose personal computer and software system to control the operations and perform the floating-point computations between timesteps. In addition, hardware and software equipment is needed to make use of the running estimates provided by the outputs of the filter. For EEG feedback, this would probably be an analog-display device; for automobile steering movements, it might be an alarm-output device triggered only when a dangerous condition is diagnosed.

Important symbols used throughout the following sections are:

$n$ discrete time-step index

$t$ time in seconds 


$\begin{array}{ll}\Delta_{t} & \text { discrete time-step size } \\ y_{n} & \text { the } n \text {th preceding time-series observation } \\ \theta & \text { frequency in radians per time-step } \\ f & \text { frequency in Hz } \\ a, b & \text { Fourier cosine and sine coefficients } \\ m, r & \text { parameters of the weight function } \\ w_{n} & \text { the weight function } \\ U, V & \text { sums of products in the least-squares problem } \\ P, Q, R & \text { sums of products in the least-squares problem } \\ \mu, \sigma & \text { parameters of the negative binomial } \\ & \text { distribution } \\ \phi & \text { target frequency in Hz } \\ x & \text { Student's } t \text { variate } \\ s & \text { spectral window spread in Hz } \\ g(x) & \text { Student's } t \text { density, unnormalized } \\ & \text { with } g(0)=1\end{array}$

\section{THEORY}

Three simple mathematical principles are important in the derivation of NEWSART filters. The first principle is the expression for the sum of the infinite geometric series,

$$
\sum z^{n}=(1-z)^{-1},
$$

for complex $z$ with $|z|<1$. In Equation 1, and throughout this paper unless indicated otherwise, summation is over the integer $n$ on the range from 0 to $\infty$. The second principle is the binomial expansion of the sum of two numbers, $p$ and $q$, raised to the $j$ th nonnegative integral power:

$$
(p+q)^{j}=\sum_{k=0}^{j}\left(\begin{array}{l}
j \\
k
\end{array}\right) p^{k} q^{j-k},
$$

in which $\left(\begin{array}{l}j \\ k\end{array}\right)=j ! /[k !(j-k) !]$ with $0 \leq k \leq j$. The third principle is the pair of Euler identities,

$$
\begin{aligned}
& \cos (\theta)=[\exp (i \theta)+\exp (-i \theta)] / 2 \\
& \sin (\theta)=[\exp (i \theta)-\exp (-i \theta)] / 2 i,
\end{aligned}
$$

where $\theta$ is real and $i=\sqrt{-1}$. These principles are used to derive the computational formulas that arise from the following least-squares rationale.

\section{The Weighted Least-Squares Criterion}

Let $\left[\ldots, y_{2}, y_{1}, y_{0}\right]$ be a sequence of quantitative measurements of some real variable, obtained at equally spaced points in time, with the time index reversed. Thus, $y_{0}$ is the most recent observation, $y_{1}$ is the one obtained immediately before $y_{0}$, and so forth. This reversed-time indexing adopts the perspective of an observer in the present looking back at past data. Notationally, it tends to simplify subscript expressions in the recurrence formulas. Let $\hat{y}_{n}$ be an approximation of $y_{n}$, based on a single pair of Fourier terms for a specific target frequency, $\theta$, with $0<\theta<\pi$. Then

$$
\hat{y}_{n}=a \cdot \cos (n \theta)+b \cdot \sin (n \theta),
$$

where the values of the two Fourier coefficients, $a$ and $b$, are to be estimated from the past data. Weighted leastsquares estimation is used, such that very old data are entered with very little weight. This is accomplished by the use of a tapered weight function that acts as a sliding timewindow. Specifically, the weight function, $w_{n}$, is defined as

$$
w_{n}=(1-r)^{m}\left(\begin{array}{c}
n+m-1 \\
n
\end{array}\right) r^{n} .
$$

In Equation 6, $m$ is a small positive integer and $r$ is real in the range $0<r<1$. The least-squares estimates of $a$ and $b$ are determined so that the residual sum of weighted squares,

$$
W=\sum w_{n}\left(y_{n}-\hat{y}_{n}\right)^{2},
$$

is minimized.

The weight function of Equation 6 was chosen for several reasons. First, it generalizes the simple geometric $(m=1)$ in a way that improves the estimates for some purposes, and it is the most mathematically convenient expression compared to similar functions. An example of a similar function is $n^{m-1} r^{n}$, which appears simpler than $w_{n}$, but leads to more complicated derivations and computational formulas. The function $w_{n}$ is of the form of the negative binomial distribution. Although there is no probabilistic interpretation here, this connection is of interest because the general properties of the function are well-known from probability theory (Feller, 1957). The negative binomial is a discrete analog of the continuous gamma or chi-square distribution, and it is the $m$-fold convolution of the simple geometric distribution.

Let $P, Q, R, U_{0}$, and $V_{0}$ be defined as

$$
\begin{aligned}
P & =\sum w_{n} \cos ^{2}(n \theta) \\
Q & =\sum w_{n} \sin ^{2}(n \theta) \\
R & =\sum w_{n} \sin (n \theta) \cos (n \theta) \\
U_{0} & =\sum w_{n} \cos (n \theta) y_{n} \\
V_{0} & =\sum w_{n} \sin (n \theta) y_{n} .
\end{aligned}
$$

Then the usual operation of setting the partial derivatives of $W$ equal to zero in Equation 7 gives the normal equations,

$$
\begin{aligned}
& U_{0}=a P+b R \\
& V_{0}=a R+b Q .
\end{aligned}
$$

This pair of equations must be solved anew for the values of $a$ and $b$ as each new time-series datum becomes available. Under conditions that can sometimes be achieved in practice, it is approximately true that $R=0$ and $P=Q=1 / 2$. However, computational formulas for $P$, $Q$, and $R$ generally are needed, as well as for $U_{0}$ and $V_{0}$.

The computational formulas are derived as if only the pair of $(a, b)$ values for a single target frequency were to be estimated. However, the intention is that running estimates can be obtained for several target frequencies (say $N$ ) concurrently. Strictly, then, the pair of normal Equations 13 and 14 should be replaced by a system of $2 \mathrm{~N}$ interdependent simultaneous equations because the estimates for different target frequencies are not generally orthogonal. However, they can be made nearly orthogonal 
by sufficient spacing of the target frequencies, and by parameter options to be discussed later. Therefore, it is assumed for approximation purposes that they are orthogonal and that the corresponding pairs of Equations 13 and 14 can be solved in parallel without cross-coupling. The more general treatment would lead to the additional computations at each time-step, of multiplying the generalized $2 N$ element $(U, V)$ vector by a $2 N \times 2 N$ element matrix that is the inverse of a generalized $(P, Q, R)$ crossproduct matrix.

\section{Evaluation of $\boldsymbol{P}, \boldsymbol{Q}$, and $\boldsymbol{R}$}

The values of $P, Q$, and $R$ are fixed, for fixed $m, \theta$, and $r$. They do not change from one time-step to the next, so their values can be computed before the start of an experiment and they need not be updated in real time. As a preliminary step in finding computational formulas for $P, Q$, and $R, \sum w_{n}$ is evaluated first. The results of that derivation are then carried over to the evaluation of $P$, $Q$, and $R$. A two-step operation is applied to both sides of Equation 1. The first step is multiplication by $z^{m-1}$, and the second step is differentiation $m-1$ times with respect to $z$. From the left side of Equation 1 it is found that

$$
\frac{\partial^{m-1}}{\partial z^{m-1}} \sum z^{n+m-1}=(m-1) ! \sum\left(_{n}^{n+m-1}\right) z^{n}
$$

From the right side,

$$
\frac{\partial^{m-1}}{\partial z^{m-1}}\left[z^{m-1}(1-z)^{-1}\right]=(m-1) !(1-z)^{-m} .
$$

Equation 16 is obtained by the use of Leibnitz's rule for the $(m-1)$ th derivative of a product. The right sides of Equations 15 and 16 must be equal, which shows that

$$
\sum\left({ }^{n+m-1}\right) z^{n}=(1-z)^{-m},
$$

and therefore that $\sum w_{n}=1$.

To find closed expressions for $P, Q$, and $R$ by the above method, a prior step is to convert $\cos ^{2}(n \theta), \sin ^{2}(n \theta)$, and $\cos (n \theta) \sin (n \theta)$ to their complex exponential forms. Then $z$ can be taken in turn as $r \cdot \exp (2 i \theta)$ and $r \cdot \exp (-2 i \theta)$ in Equation 17 to obtain components of the sums. These components have complex values, but they pair up in ways that yield real computational formulas. In particular, let

$$
\begin{aligned}
& A=\sum_{j=0}^{m}\left(\begin{array}{l}
m \\
j
\end{array}\right)(-1)^{j} r^{j} \cos (2 j \theta), \\
& B=\sum_{j=0}^{m}\left(\begin{array}{c}
m \\
j
\end{array}\right)(-1)^{j} r^{i} \sin (2 j \theta),
\end{aligned}
$$

and

$$
C=(1-r)^{m}\left[1-2 r \cos (2 \theta)+r^{2}\right]^{-m} .
$$

Then the computational formulas for $P, Q$, and $R$ are:

$$
P=(1+A C) / 2,
$$

$$
Q=(1-A C) / 2
$$

and

$$
R=-B C / 2
$$

\section{Recurrence Relations for $\boldsymbol{U}_{\mathbf{0}}$ and $\boldsymbol{V}_{\mathbf{0}}$}

Unlike $P, Q$, and $R, U_{0}$ and $V_{0}$ do change as each new datum becomes available in the running-time series. The problem in trying to use Equations 11 and 12 directly is that the whole infinite sequence of $y_{n}$ values must be shifted at each new step, relative to the sequence represented by the other factors under the summation sign. Fortunately, there is a recursive way of calculating this shift with just a few operations.

The computations of the current values of $U_{0}$ and $V_{0}$ involve older values, so let

$$
U_{k}=\sum w_{n} \cos (n \theta) y_{n+k}
$$

and

$$
V_{k}=\sum w_{n} \sin (n \theta) y_{n+k},
$$

with $k$ being a nonnegative integer. As before, it is useful to develop a strategy without the trigonometric expressions first, and then modify it as needed. Consider the moving averages, $M_{k}$, of $y_{n}$, defined as

$$
M_{k}=(1-r)^{m} \sum\left({ }^{n+m-1}\right) z^{n} y_{n+k} \text {. }
$$

There are several ways to compute the value of $M_{0}$ from $y_{0}$ and a few $y_{k}$ and $M_{k}$ values for $k>0$. The method used here, because of its efficiency and convenience of form, is

$$
M_{0}=(1-r)^{m} y_{0}-\sum_{k=1}^{m}\left(\begin{array}{c}
m \\
k
\end{array}\right)(-1)^{k} z^{k} M_{k} .
$$

A general proof (not presented here) of Equation 27 can be constructed by the use of negative binomial coefficients (Feller, 1957) that are based on a generalization of $(k)$ for negative $j$. It is not hard to show, without that generalized definition, that Equation 27 holds for particular small values of $m$. The use of Equation 27 requires saving the most recent $M_{0}, M_{1}, \ldots M_{-1}$ values, which become $M_{1}$, $M_{2}, \ldots M_{m}$ on the next time-step. Old values of $y_{n}$ are not needed.

The substitution of $r \cdot \exp (i \theta)$ and $r \cdot \exp (-i \theta)$ for $z$ in Equation 27, followed by manipulations using the Euler identities of Equations 3 and 4, gives these recurrence relations for $U_{0}$ and $V_{0}$ :

$$
\begin{aligned}
U_{0}= & (1-r)^{m} y_{0} \\
& -\sum_{k=1}^{m}\left(\begin{array}{l}
m \\
k
\end{array}\right)(-1)^{k} r^{k}\left[\cos (k \theta) U_{k}-\sin (k \theta) V_{k}\right]
\end{aligned}
$$

and

$$
V_{0}=-\sum_{k=1}^{m}\left(\begin{array}{c}
m \\
k
\end{array}\right)(-1)^{k} r^{k}\left[\sin (k \theta) U_{k}+\cos (k \theta) V_{k}\right] .
$$

For practical computations in real time, vectors of 
$\left(\begin{array}{c}m \\ k\end{array}\right)(-1)^{k} r^{k} \cos (k \theta)$ and $\left(\begin{array}{c}m \\ k\end{array}\right)(-1)^{k} r^{k} \sin (k \theta)$ can be computed and stored in memory before the startup of the time series. Then the number of multiplications per time-step, in updating the values of $U_{0}$ and $V_{0}$, is $4 m+1$. The $U$-V shifting operation at each step $\left(U_{k+1} \leftarrow U_{k}\right.$ and $V_{k+1} \leftarrow V_{k}$, for $K=0,1, \ldots m-1)$ can be done with cycling pointers to avoid moving the data in memory.

\section{IMPLEMENTATION}

There are several points that require some attention in applying the theoretical results to the real-time analysis of time-series data. A number of these points carry over from principles that are familiar in applications of the ordinary discrete Fourier transform to fixed-length record segments (Blackman \& Tukey, 1958; Emerson, 1983; Kaplan, 1983; Yost, Cooper, \& Bremner, 1983), but others arise as special issues unique to this recursive type of spectral estimation.

\section{Scaling}

The theoretical representations of time and frequency, $n$ and $\theta$, are on operational scales of measurement. For practical measurement in units of seconds and hertz, let $t$ be time and $f$ be frequency. An early step in applying the theory is to adopt an elementary time unit, $\Delta_{t}$, which is the length of time between successive discrete observations. Then $t=n \Delta_{t}$. Also, the theoretical frequency, $\theta$, is in radians per time-step. The change to $f$ in hertz is given by $f=\theta /\left(2 \pi \Delta_{t}\right)$. However, using the theoretical results usually involves the substituting $2 \pi f \Delta_{t}$ for $\theta$ in the formulas.

\section{The Nyquist Frequency and Aliasing}

As with applications of Fourier analysis to discrete records of fixed length, the Nyquist frequency is $1 /\left(2 \Delta_{t}\right) \mathrm{Hz}$, or $\pi$ radians per time-step. A stage of analog low-pass filtering must precede the data digitization if aliasing is to be avoided from frequencies above $1 /\left(2 \Delta_{t}\right)$. NEWSART filtering gives a bell-shaped power-gain window centered at the target frequency. However, that window is replicated in an infinite periodic way at frequencies above $1 /\left(2 \Delta_{t}\right)$. Prior low-pass analog filtering is virtually the only way to block out the contributions from the alias windows above $1 /\left(2 \Delta_{t}\right)$ (Blackman \& Tukey, 1958). Moreover, with NEWSART filtering in particular, estimates at frequencies less than but near the Nyquist frequency have undesirable irregularities. Therefore, it may be desirable to adjust the low-pass analog filter to provide some attenuation at and near the Nyquist frequency.

It is also highly desirable, for any kind of digital realtime filtering, to include a prior stage of high-pass ana$\log$ filtering to eliminate contributions from frequencies at and near 0 .

\section{General Points of Design Rationale}

The general effects of several parameters are qualitatively apparent from the theoretical results. More detailed information is available by numerical investigation using the two test programs shown in the program listings. The decay constant, $r$, determines the asymptotic rate of decay of the effects of old data on the current spectral estimates. However, the shape of the weight function for data that are not very old depends strongly on the value of $m$. As $m$ becomes larger, the shape of this sliding timewindow becomes more nearly symmetrical and bell shaped (Figure 1). One of the main reasons for not always taking $m=1$ is the rakish attack of the geometric function with its sharp peak at $n=0$. From experience with fixedrecord Fourier analysis, it is generally known that a timewindow should be tapered toward zero on both ends, to reduce spurious effects on the frequency estimates. Increasing $m$ will achieve gentleness in the leading edge of the time-window with NEWSART filtering. One result of increasing $m$ that is quite clear from numerical studies is that it reduces the beat-frequency oscillations, which can be rather severe with $m=1$. These are oscillations of the power estimates at frequency $|f-\phi|$, where $\phi$ is the target frequency and $f$ is the frequency of a steady sinusoidal input signal. Theoretically, there should be a component at $f+\phi$ too, but the one at $|f-\phi|$ is most notable in the numerical simulations. It is evident from Equations 11 and 12 that the estimation of $U$ and $V$ is similar to amplitude modulation in which a steady carrier (at frequency $\phi$ ) is modulated by a second signal (at frequency $f$ ) to generate heterodyne products at the sum and difference frequencies. In radio technology, some of the heterodyne products are of great value and interest, but they are unwanted in most plausible applications of NEWSART.

Increases in $m$ and $r$ alike increase the settling time, or lag, of the appearance of changes in the output estimates behind the times of the antecedent changes in the input time series. Likewise, increases of $m$ and $r$ have

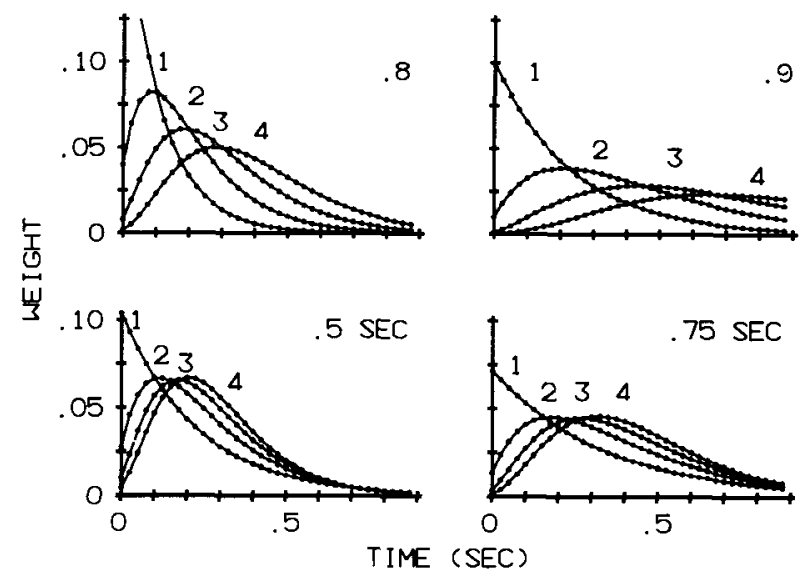

Figure 1. Variations of the shape of the negative binomial weight function as a function of changes in the values of the parameters $m$ and $r$. In the top two graphs, $m$ is varied while $r$ is held fixed at .8 (left) and .9 (right). In the bottom graphs, $m$ is varied with compensating variations of $\boldsymbol{r}$ to achieve the fixed $90 \%$ settling times of $.5 \mathrm{sec}$ (left) and $.75 \mathrm{sec}$ (right). In all cases, $\Delta_{t}=.025 \mathrm{sec}$, and the dots are plotted at integral multiples of this interval. Connecting straight lines are included for clarity. 


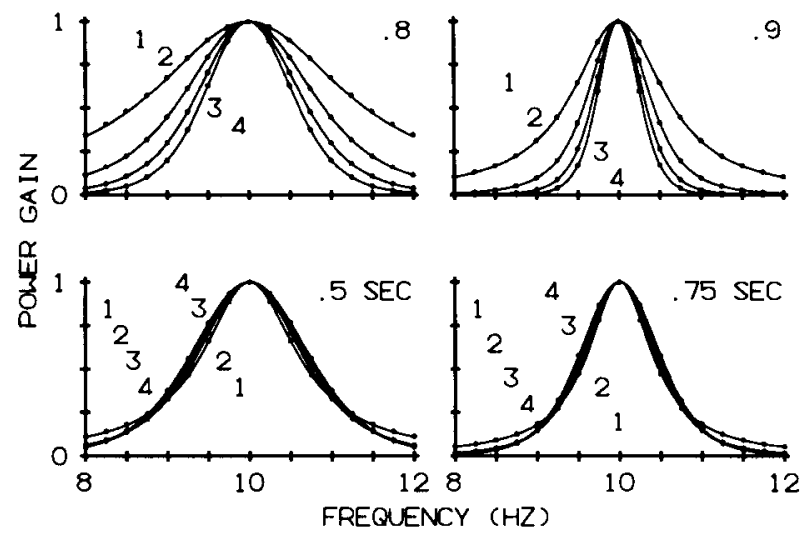

Figure 2. Average spectral windows obtained with the use of the weight functions shown in Figure 1 . In the top two graphs, the windows become lower, except at the center point, with increasing $m$. In the bottom graphs, the windows become lower in the tails with increasing $m$, but higher at points near the center. The dots are the averages given from the simulation-test program, and the smooth curves are the Student's $t$ densities from Equations 31-33. Parameter values were as in Figure 1, with a target frequency of $10 \mathrm{~Hz}$.

the general effect of narrowing the frequency-bandpass window about the target frequency (Figure 2). A third effect, related to the second, is that the estimated quantities become more nearly orthogonal. This is partly reflected by the expression for $R$ in Equation 23. Note that it is possible, when estimating a single target frequency, to make $R$ vanish identically. This occurs if $\theta$ is taken as $\pi / 2$, which is the midpoint of the range of frequencies that can be estimated. When there is more than one target frequency, this condition cannot be forced identically for all of them, but it is approximated closely if $m$ is fairly large and if the target frequencies are not very near the ends of the range. One further side-effect of taking $m$ or $r$ relatively large is that the values of $P$ and $Q$ become more nearly equal to $1 / 2$.

\section{Settling Time}

To some extent, the effects of $m$ and $r$ are similar, which makes it difficult to assess the effects of either parameter when both are varied. However, there is a way to control this tradeoff by using a settling criterion, instead of $r$, as one of the two free parameters. This scheme is worked out here for a $10 \%$ settling-time criterion with the parameter $n 10$ being the value of $n$ such that the tail of $w_{n}$ sums to .10 beyond $n 10$. Thus, data older than $n 10$ (in units of $n$ ) enter into the present spectral estimate with a weight of $10 \%$ relative to the total.

The user specifies the values of $m$ and $n 10$, instead of $m$ and $r$, and then calculates the value of $r$ needed to satisfy the $10 \%$ criterion. The main problem with this scheme is that it is not easy to find the precise value of $r$ that satisfies the $10 \%$ criterion in a direct way. However, close approximations are available from Table 1 , which was constructed using a computer program that finds the exact desired values of $r$ but is not convenient for ordinary use. For values of $n 10$ not listed, an approximation can be used. The following method is usually more accurate than simple linear interpolation, and can also be used for extrapolation. Let $n_{2}$ be the desired value of $n 10$, and $n_{1}$ be the nearest value to it that appears in the table. Let $r_{2}$ and $r_{1}$ be the corresponding values of $r$ for the same value of $m$. Then the approximation to $r_{2}$ is

$$
r_{2}=n_{2} /\left[n_{2}+n_{1}\left(1-r_{1}\right) / r_{1}\right] \text {. }
$$

This approximation is derived from the observation that $n(1-r) / r$ varies gradually across a given row in Table 1 for fixed $m$, as $n$ and $r$ change.

A useful alternative is a normal approximation that gives $n 10$ as a function of $m$ and $r$. It is based on the known mean and standard deviation of the negative binomial, $\mu=m r(1-r)^{-1}$ and $\sigma=\sqrt{m r}(1-r)^{-1}$ (Parzen, 1962). The approximation, $n 10=\mu+1.28 \sigma$, gives a second-degree equation in $r$ that can be solved for $r$ with the quadratic

Table 1

Values of $r$ Needed for Specified Settling Times by the $10 \%$ Criterion

\begin{tabular}{|c|c|c|c|c|c|c|c|c|}
\hline \multirow[b]{2}{*}{$m$} & \multicolumn{8}{|c|}{$n 10$} \\
\hline & 5 & 10 & 20 & 40 & 80 & 160 & 320 & 640 \\
\hline 1 & .68129 & .81113 & .89615 & .94539 & .97197 & .98580 & .99285 & .99641 \\
\hline 2 & .54794 & .71250 & .83441 & .91052 & .95339 & .97620 & .98797 & .99396 \\
\hline 3 & .46179 & .64022 & .78481 & .88093 & .93715 & .96768 & .98361 & .99174 \\
\hline 4 & .40058 & .58302 & .74246 & .85443 & .92220 & .95972 & .97950 & .98966 \\
\hline 5 & .35422 & .53603 & .70533 & .83016 & .90815 & .95214 & .97556 & .98765 \\
\hline 6 & .31772 & .49649 & .67226 & .80768 & .89482 & .94485 & .97174 & .98569 \\
\hline 7 & .28817 & .46265 & .64248 & .78668 & .88209 & .93779 & .96802 & .98378 \\
\hline 8 & .26373 & .43329 & .61547 & .76695 & .86986 & .93093 & .96437 & .98190 \\
\hline 9 & .24316 & .40754 & .59079 & .74836 & .85809 & .92424 & .96080 & .98005 \\
\hline 10 & .22559 & .38475 & .56813 & .73077 & .84674 & .91772 & .95729 & .97823 \\
\hline 11 & .21041 & .36443 & .54724 & .71409 & .83575 & .91133 & .95384 & .97643 \\
\hline 12 & .19716 & .34619 & .52790 & .69823 & .82511 & .90508 & .95043 & .97465 \\
\hline 13 & .18549 & 32971 & .50993 & .68312 & .81480 & .89894 & .94707 & .97289 \\
\hline 14 & .17513 & .31476 & .49318 & .66870 & .80478 & .89292 & .94375 & .97114 \\
\hline 15 & .16587 & .30111 & .47753 & .65493 & .79505 & .88701 & .94047 & 96941 \\
\hline 16 & .15755 & .28462 & .46288 & .64174 & .78558 & .88120 & .93723 & .96770 \\
\hline
\end{tabular}


formula. Both approximations improve with increasing $n 10$, but they are remarkably good even for small $n 10$. The approximation based on Equation 30 is more handy for estimating the value of $r$ needed to achieve a given value of $n 10$, whereas the normal approximation is convenient to estimate $n 10$ from given values of $m$ and $r$.

Settling time is easier to interpret as one of the free parameters than is $r$. From a practical standpoint, settling time is an index of how long the estimation process should be allowed to run after startup, so that artifactual startup transients will be significantly attenuated. In connection with conditioning theory in biofeedback applications, settling time is an approximate index of the length of the delay of feedback. In connection with research applications in which a statistical summary of the running estimates is needed for later analysis, decimation can be used with the selection of only every $n 10$ th estimate to be included in the summary. The successive estimates in this decimated summary will usually have considerably lower autocorrelation values than those in the full sequence of estimates, and might be treated as statistically independent for some purposes.

\section{Power-Gain Windows}

Many of the features of a NEWSART filter that are of interest in evaluating its performance are revealed by the power-gain window obtained for one target frequency at a time. This window is obtained by feeding a single-unit amplitude sinusoid to the filter at each of a number of test frequencies arrayed around the target frequency. For a given input test frequency, the estimates are ignored after startup for a number of steps somewhat greater than $n 10$, and power estimates are then observed over a lengthy sequence of time-steps. This sample yields three statistics that are of interest: the average, the maximum, and the minimum of the power estimates. The differences between the maxima and minima indicate the magnitudes of the beat oscillations, and the averages give a summary picture of the shape of the power-gain window. Test Program 2 performs such computations.

\section{Frequency Selectivity}

An important general principle was discovered by running Test Program 2 in a large number of cases that reveals almost all that one might wish to know about the frequency selectivity of a NEWSART filter. This principle applies best under favorable estimation conditions in which the power-gain window is not very close to either end of the frequency range.

At first glance, the average power-gain windows appeared normal (Gaussian) in shape. However, closer inspection and attempts to fit the normal density revealed that the window tails were too high. This suggested Student's $t$ density function, which was found to provide astonishingly precise fits. Moreover, the proper value of the $d f$ parameter of Student's distribution was determined with no ambiguity to be $2 m-1$. This left a free-scale parameter, $s$, to be estimated numerically. Let $x$ be the variate of Student's distribution ( $x$ rather than $t$ because $t$ is used here for time), and let $f$ be the test frequency and $\phi$ the target frequency in hertz. Then

$$
x=(f-\phi) / s .
$$

Numerical estimates of $s$ in various cases revealed that

$$
s=(1-r)\left[2 \pi \Delta_{t} \sqrt{r(2 m-1)}\right]^{-1}
$$

to an extremely close approximation. To summarize these findings, let $g(x)$ be an ideal continuous power-gain function that is approximated discretely by the numerical estimates from Test Program 2. Substituting $2 m-1$ for the $d f$ parameter in the expression for Student's density (Kendall \& Stuart, 1963, p. 257), and neglecting the normalizing factor, which is not needed, yields

$$
g(x)=\left[1+x^{2} /(2 m-1)\right]^{-m} .
$$

With $x$ and $s$ as defined in Equations 31 and 32, Equation 33 gives excellent matches to the average power-gain estimates from Test Program 2.

It is both curious and convenient that the negative binomial time-window generates a spectral window of the form of Student's density function. The curiosity is that Student's density obviously does not fit well in extreme cases in which the target frequency is very near one end of the frequency range, but it fits so well otherwise.

The convenience is that some form of Student's $t$ table may be found in many statistics texts and reference books that provide proportional areas under the curve in selected ranges. The scale factor, $s$, is the most direct single index of the width of the spectral window along the frequency scale. For preliminary planning, as well as for an overall understanding of the effects of parameter values, it is useful to note that the NEWSART settling time in seconds is nearly inversely proportional to $s$ in hertz as given in Equation 32, without regard to the particular $m, r$, and $\Delta_{t}$ values that produce that value of $s$. For $m$ not larger than about 5 , settling time is given roughly as $.36 / \mathrm{s} \mathrm{sec}$, varying only in the range from about $.3 / s$ at $(m, n 10)=(1,5)$ to $.4 / s$ at $(m, n 10)=(5,80)$. The bottom two graphs in Figure 2 illustrate the approximate invariance of the spectral-window width under variations of $m$ and $r$, with settling time held constant.

Another approximation to the average spectral window is $g^{\prime}(x)$ given by

$g^{\prime}(x)=(1-r)^{2 m}\left[1-2 r \cdot \cos \left(2 \pi \Delta_{t} s \cdot x\right)+r^{2}\right]^{-m}$.

This is the squared magnitude of the discrete Fourier transform of the negative binomial time-window. Its theoretical basis is more direct and it correctly shows the periodicity at frequencies above the Nyquist frequency. Equation 33, which is derivable as the squared magnitude of the continuous Fourier transform of a gamma distribution, does not show this periodicity. For reasonably favorable conditions of estimation, however, $g(x)$ typically gives values that agree to two or more decimal places with those of $g^{\prime}(x)$, and Student's $t$ tables are readily available for proportional areas under $g(x)$, so $g^{\prime}(x)$ seems to 
have little practical advantage. An identity that is of theoretical interest is that the substitution of $1-\theta^{2} / 2$ for $\cos (\theta)$ in the expression for $g^{\prime}(x)$ gives $g(x)$. This quadratic approximation to $\cos (\theta)$ is the MacLaurin expansion truncated after the first two nonzero terms, and it is a very good approximation for small $|\theta|$. Thus, Equations 31-33 have an indirect theoretical basis beyond the empirical curve fitting by which they were discovered.

\section{Design Steps}

When running estimates are to be obtained with several target frequencies concurrently, there is no theoretical feature of NEWSART that requires that the parameter pair $(m, n 10)$ be the same for the different target frequencies. In addition, it is clear that there is little or no speed advantage in the real-time computations in using the same parameter pair with the different frequencies. However, it is often desirable for the estimates at the different target frequencies to have similar sampling characteristics, settling times, and spectral window shapes, and those goals are achieved by using the same $(m, n 10)$ pair. With a single $A / D$ converter, the use of different values of $\Delta_{t}$ for the different target frequencies would introduce practical complications in the analog preprocessing and in the programming of the $\mathrm{A} / \mathrm{D}$ conversions. Therefore, the following illustrations of design planning all assume that the same $\left(m, n 10, \Delta_{t}\right)$ set is used at the different target frequencies. With more than one A/D channel, each with its own target frequency, the same principles would apply but with each channel possibly having its own analog preprocessing and $\left(m, n 10, \Delta_{t}\right)$ set.

There are a number of decisions to be made in the design of an experiment, and some of these decisions impose constraints on others. There is a fairly logical sequence of steps that tends to reduce wasted effort but does not eliminate trial and error altogether. There are three main design steps, with a number of substeps. The first step is to determine the number of target frequencies and their identities, the second step is to determine the discrete sampling rate, and the third step is to determine suitable values of the parameters, $m$ and $r$.

Two test programs are presented for use in this procedure. These programs compile directly with Datalight $\mathrm{C}$ on machines that use MS-DOS. They compile with Microsoft and Turbo $\mathrm{C}$ as well, but the clock routine (Emerson, 1988) must be compiled and linked with the first test program because it is not in the Microsoft and Turbo libraries. It is important that the test programs be compiled with the same language, implementation, and computer for the timing tests that will be used for conducting the actual experiment.

Step 1: Determination of the target frequencies. This decision is made primarily from the goals of the research to be performed. It is not necessary that the number of target frequencies be an integral power of 2 , as is required by some forms of the FFT, and the frequencies are not restricted to a discrete set of points that are equally spaced. However, speed limitations make it generally desirable to use as few target frequencies as possible, and it is not feasible with contemporary personal computers to choose target frequencies too high into the auditory range. In addition, frequencies that are too closely spaced will yield nonorthogonal estimates, and it is not wise to try to estimate simultaneously at target frequencies that differ by a large multiplicative factor, unless a long settling time is acceptable.

Step 2: Determination of the needed sampling rate. Consider the lowest and highest target frequencies, and call these $\phi_{1}$ and $\phi_{2}$. Both of these must be in the range from 0 to $1 /\left(2 \Delta_{t}\right)$, and it is desirable that they be approximately centered. Therefore, take $\left(\phi_{1}+\phi_{2}\right) / 2=1 /\left(4 \Delta_{t}\right)$, and solve for $\Delta_{t}$, which yields $\Delta_{t}=1 /\left[2\left(\phi_{1}+\phi_{2}\right)\right]$. This is merely an ideal value for $\Delta_{t}$; other constraints may force some deviation from it.

Step 3: Determination of the needed values of $m$ and $r$. This process may require a few cycles using the test programs before satisfactory results are obtained. The steps in this cycle are: (a) specify a tentative settling time in seconds, and divide this by $\Delta_{t}$ to obtain $n 10$; (b) choose a tentative value of $m$, perhaps $m=5$ to start; (c) using Table 1 and Equation 30, determine the value of $r$ from the given tentative values of $m$ and $n 10$; (d) run Test Program 1 to see if it will keep up with the sampling rate given these parameter values. If it will not, reduce $m$ or the number of target frequencies; (e) examine $R$ for each of the target frequencies using either of the test programs. It is desirable that $R=0$, to a near approximation $(|R|<.01$ for example), so that Equations 13 and 14 need not be solved simultaneously. If $R$ is not close to 0 , try increasing either $m$ or the settling time; (f) examine the power-gain windows for serious overlap and beat-oscillation magnitudes for the various target frequencies using Equations 31-33 and Test Program 2. If the window overlap is small, then the estimates will be nearly orthogonal.

Consider an exception in Step 3e. Other constraints may prevent achieving $R=0$, approximately. An alternative is to solve Equations 13 and 14 simultaneously at each time-step rather than making the assumption that $R=0$. Test Program 1 provides the option of making the speed test with or without these extra computations.

These three planning steps may optionally be augmented by a fourth step to increase the reliability of spectral estimates without affecting the spectral windows determined by the choices in the first three steps. The rationale of the fourth step follows.

\section{Stability of Estimates and NEWLIL Filtering}

An extension of NEWSART filtering is available to decrease the variability of spectral estimates of signals that are accompanied by noise. The extension is to add a succeeding stage of NEWLIL (NEgative binomial Weighted LInear Lowpass) filtering based on the formal characteristics of Equation 27, with $z$ replaced by $r$ and $y_{0}$ replaced by $a^{2}+b^{2}$. For convenience, the NEWLIL geometricdecay constant can be taken to be the same as $r$ in the 
NEWSART filter. Let the NEWLIL parameter corresponding to $m$ be $m^{\prime}$, and let $h_{0}$ be the output of the NEWLIL stage, with $a^{2}+b^{2}$ being the output of the NEWSART stage. Taking $m^{\prime}=1$ for illustration, the recurrence relation for $h_{0}$ is

$$
h_{0}=(1-r)\left(a^{2}+b^{2}\right)+r \cdot h_{1} .
$$

It is clear that the additional NEWLIL stage leaves the shape and width of the NEWSART average powergain window (Equations 31-33) entirely unchanged. The NEWLIL stage provides an escape from the reciprocity of the widths of the time and spectral windows that is characteristic of the NEWSART tradeoff. NEWLIL trades longer settling time for more reliable spectral estimates, whereas NEWSART trades longer settling time for narrower spectral windows. Both options are useful in the context of off-line spectral analysis of finite-length data records, as was explained by Blackman and Tukey (1958, pp. 93-95).

The combined settling time for a NEWSART stage with parameter $m$, followed by a NEWLIL stage with parameter $m^{\prime}$, is the same as that for a NEWSART filter with $m$ replaced by $m+m^{\prime}$, other things being equal.

\section{NEWLIB for Speedier Computations}

Yet another extension of NEWSART theory has been explored because a reviewer suggested that the output of a linear bandpass filter be rectified (squared) and smoothed with a succeeding NEWLIL stage, to accomplish the same effect as that from NEWSART, with some possible savings of computation time.

There are hundreds of linear bandpass filters and dozens of families to choose from (Kaiser, 1966), so the main problem is to choose one that is closely comparable to NEWSART. The NEWLIB (NEgative binomial Weighted LInear Bandpass) filter, described here, appears to be as closely comparable to NEWSART as any other filter. NEWLIB has the same parameters and its characteristics are quite similar to those of NEWSART. The weighted least-squares criterion of NEWSART is not imposed, and the derivation, the details of which are not presented here, proceeds instead from the NEWLIL filter by Broome's (1966) method of converting a linear low-pass filter to a linear bandpass filter. The result is

$$
B_{0}=\sum_{k=0}^{m} c_{k} y_{k}-\sum_{k=1}^{2 m} d_{k} B_{k} .
$$

In Equation 35, $B_{0}$ is the present NEWLIB output, $B_{k}$ for $k>0$ are the past outputs, and $y_{k}$ are the corresponding inputs. The constant coefficients, $c_{k}$ and $d_{k}$, are given by

$$
c_{k}=2(-1)^{k} r^{k}(1-r)^{m}\left(\begin{array}{c}
m \\
k
\end{array}\right) \cos (k \theta)
$$

and

$$
d_{k}=(-1)^{k} r^{k} \sum_{j=0}^{k}\left(\begin{array}{c}
m \\
j
\end{array}\right)\left(\begin{array}{c}
m \\
k-j
\end{array}\right) \cos [(k-2 j) \theta],
$$

where $\theta$ is the desired center of the frequency passband.
The $c$ and $d$ vectors are computed numerically before the real-time processing begins, so the real-time computations involve only those multiplications and additions shown in Equation 35, together with saving the old $y_{k}$ and $\boldsymbol{B}_{k}$ values and shifting from one time-step to the next $\left(y_{k+1} \leftarrow y_{k}\right.$ and $B_{k+1} \leftarrow B_{k}$, for $\left.k=0,1, \ldots, 2 m-1\right)$. The timeshifting operations can be programmed with circular modulo pointers to avoid moving the data in memory.

A slight inconvenience of this method of NEWLIB computations is that it requires a numerical estimation of the normalizing factor, whereas the NEWSART normalization is accomplished with $P, Q$, and $R$, the values of which are precomputed from Equations 21-23. However, this normalization is not prohibitive because a test program, with the test frequency equal to the target frequency, can be run in advance of experimental data collection. The average power estimated under this condition can be used as the divisor of subsequent real-time experimental estimates so that the resulting power-gain is unity at the target frequency. Such normalizing constants observed under test conditions had values between .5 and 1 , and approached .5 as $m$ or $r$ was taken large.

For the speed comparisons between NEWLIB and NEWSART, it is convenient to use the easily countable number of multiplications and divisions, with the number of additions and subtractions tending to be nearly proportionate. With NEWLIB, the number of multiplications in the primary updating process is $3 m+1$; the succeeding squaring operation requires one more multiplication, and the normalizing yet another, for $3 m+3$ in all. For NEWSART, the primary updating requires $4 m+1$, the normalizing requires 6 more multiplications (not assuming that $R=0$ ), and the squaring of 2 more, for $4 m+9$ per time-step. For power estimates, with NEWLIB, the following NEWLIL smoothing stage is more crucial than with NEWSART, but would usually be desired for noise suppression in either case. From these figures, it is estimated that the NEWLIB computations would be executed at best in about half the time of those of NEWSART for small $m$, and in about three-fourths of the time for large $m$.

The average power spectral-window estimates from NEWLIB and NEWSART were surprisingly similar, but were noticeably different in some cases. The NEWSART windows were more invariant under changes in the location of the target frequency within the range of estimable frequencies, and were mcre closely approximated by Student's $t$ function (see Figure 4, for example). However, these differences tended to be rather small except when the target frequency was near either 0 or the Nyquist frequency.

Another apparent advantage of NEWSART is illustrated in Figure 3, in which the test data indicate that more NEWLIL smoothing would be needed by NEWLIB in order to achieve the same smoothness obtained from NEWSART, and this would lead to a longer settling time.

NEWLIB can be useful in cases in which test runs indicate that NEWSART is almost, but not quite, fast enough. NEWLIB has exactly the same parameters as 
NEWSART, and their effects are essentially the same in the two cases, so most of the planning for an application of NEWSART would carry over if NEWLIB were used instead.

\section{A Planning Example}

Suppose that a researcher wants to estimate at three target frequencies, 3,9 , and $15 \mathrm{~Hz}$, with fairly broad spectral windows but with not much overlap between them. Suppose also that a settling time of about $.5 \mathrm{sec}$ is desired. The spectral-window width can be specified by the criterion that the interval between $\phi-s \cdot x$ and $\phi+s \cdot x$ contains $90 \%$ of the area under the power-gain window. With $s \cdot x$ between about 2 and $3 \mathrm{~Hz}$, the windows will be fairly broad, and the overlap will still be small since the spacing between adjacent target frequencies is $6 \mathrm{~Hz}$. In this example, the achievement of an $s \cdot x$ value between 2 and $3 \mathrm{~Hz}$ requires a few trials and corrections.

The discrete time-step is $\Delta_{t}=1 /[2(3+15)]=.0278 \mathrm{sec}$, or some near approximate figure that is convenient. Then $n 10=.5 / .0278=18$, which we round to 20 to avoid interpolations in the early rough planning. The suggestion of trying $m=5$ gives $r=.70533$ from Table 1 , and $s=.6696$ with $9 d f$, from Equation 32. From a $t$ table,

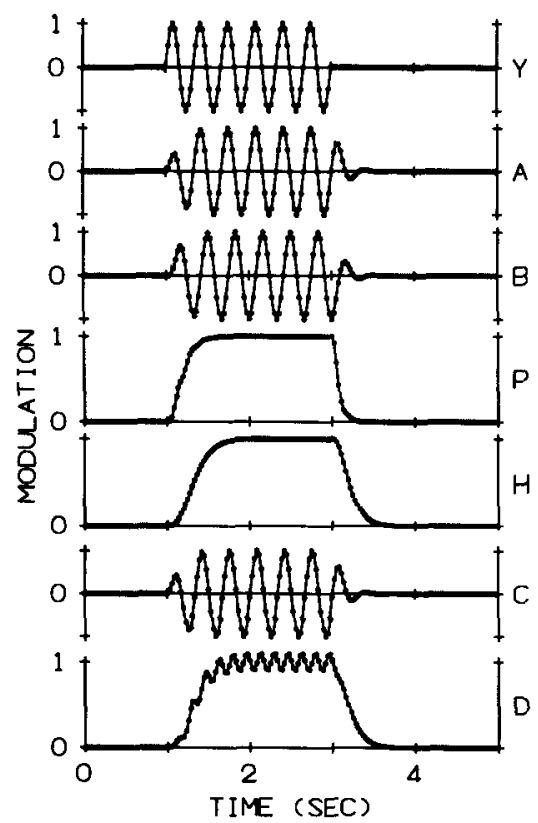

Figure 3. Time-course of estimates from NEWSART $(\mathbf{A}, \mathbf{B}, \mathbf{P}, \mathbf{H})$ and NEWLIB $(C, D)$ in response to an input signal $(Y)$ that remains constant at 0 for $1 \mathrm{sec}$, is then modulated with a unit-amplitude 3-Hz sinusoid for $2 \mathrm{sec}$, and is then held constant for $2 \mathrm{sec}$. The target frequency was $3 \mathrm{~Hz}$, with $m=2, m^{\prime}=2, \Delta_{z}=1 / 36$, and $r=.72181$. These values give a combined settling time of $.5 \mathrm{sec}$ for the traces shown in $\mathbf{H}$ and $\mathbf{D}$. The dots are plotted at integral multiples of the time-increment, and straight connecting lines are added for clarity. Traces $A$ and $B$ show the cosine and sine coefifient estimates $a$ and $b$; Trace $P$ shows $a^{2}+b^{2}$; Trace $H$ is the result of putting the $a^{2}+b^{2}$ signal through the succeeding NEWLIL stage with $m^{\prime}=2$; Trace $C$ shows the direct NEWLIB output; and Trace D shows the result of squaring that output and putting the result through the succeeding NEWLIL stage with $m^{\prime}=2$.

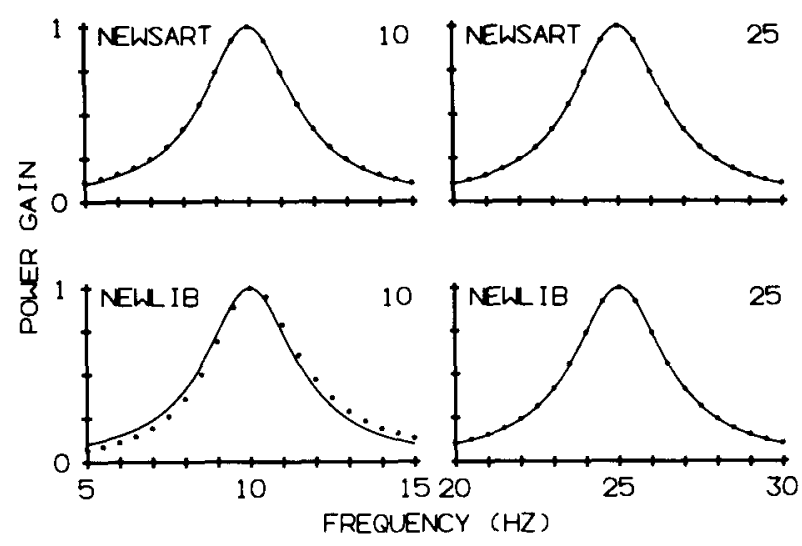

Figure 4. Comparison of average spectral power-windows from NEWSART (top) and NEWLIB (bottom) for target frequencies off center at $10 \mathrm{~Hz}$ (left) and centered at $25 \mathrm{~Hz}$ (right) in the range of estimable frequencies. Parameter values were $\Delta_{r}=.01 \mathrm{sec}, m=1$, $m^{\prime}=0$, and $r=.9$. The dots are the averages from running-test programs, and the smooth curves are the Student's $t$ densities from Equations 31-33.

the $10 \%$ two-tail critical value is $x=1.833$, so $s \cdot x=1.227$, which is not in the desired range between 2 and $3 \mathrm{~Hz}$. Therefore, the plan must be changed.

Let us try $m=2$ instead of $m=5$. Then $r=.83441$ from Table 1 , and $s=.599$ with $3 d f$. The $10 \%$ critical value with $3 d f$ is 2.353 , so $s \cdot x=1.41$, which is only a small move toward the goal. It seems that more radical change is needed.

A more drastic change is to decrease the settling time from .5 to $.25 \mathrm{sec}$, which reduces $n 10$ from 20 to 10 , approximately. Then $m=2$ gives $r=.7125$ from Table 1 , and $s=1.126$ with $3 \mathrm{df}$, making $s \cdot x=2.65 \mathrm{~Hz}$, which is satisfactory according to the stated requirement that $s \cdot x$ be between 2 and $3 \mathrm{~Hz}$. However, the settling time is shorter than necessary, so we add a stage of NEWLIL filtering, which lengthens the settling time with the bonus effect of decreasing both the random variability of the estimates and the beat oscillations. A good choice of the NEWLIL parameter value is $m^{\prime}=2$. The normal approximation for settling time with $m+m^{\prime}=4$ and $r=.7125$ gives a value of 17.4 for $n 10$, or $.485 \mathrm{sec}$. In summary, these parameter values seem adequate for the specified conditions: $\Delta_{t}=.0278, r=.7125, m=2$, and $m^{\prime}=2$. The $10 \%$ settling time is about $.485 \mathrm{sec}$ and the $90 \% \mathrm{spec}-$ tral intervals are approximately from $\phi-2.65 \mathrm{~Hz}$ to $\phi+2.65 \mathrm{~Hz}$.

Numerical tests of the spectral window shapes using Test Program 2 indicated that the above planning was fairly accurate, though there was mild asymmetry of the windows at $\phi=3 \mathrm{~Hz}$ and $\phi=15 \mathrm{~Hz}$ due to the nearness to the limits of the frequency range $(0$ and $18 \mathrm{~Hz})$. In addition, the beat oscillations were greater than normal with test frequencies near these limits. These irregularities might have been expected, since the intended $90 \%$ spectral intervals extend nearly to the limits of the frequency range- $.35 \mathrm{~Hz}$ for $\phi=3 \mathrm{~Hz}$ and $17.65 \mathrm{~Hz}$ for $\phi=15 \mathrm{~Hz}$. Irregularities associated with range-truncation 
effects would be attenuated somewhat by the prior stage of analog bandpass filtering if it rejects energy near 0 and the Nyquist frequency.

Tests with $m^{\prime}=0$ (no succeeding NEWLIL stage) and $m^{\prime}=2$ showed that the NEWLIL stage reduced the beat oscillations considerably. At $\phi=3$ and $f=4$, for example, the difference between the maximum and minimum power estimate was .220 for $m^{\prime}=0$, but only .014 for $m^{\prime}=2$. Figure 5 illustrates similar effects of a succeeding NEWLIL stage in another case. Average power estimates with $m^{\prime}=0$ were almost identical to those with $m^{\prime}=2$, which is predictable a priori.

\section{Examples of Timing Limits}

The data of Table 2 were obtained from running Test Program 1 with $m=1, m^{\prime}=0$, and a single target frequency, with the assumption that $R=0$. Thus, these data are the absolute minima of the size of $\Delta_{t}$ for this combination of computer and compiler. The operating system in all cases was MS-DOS. Note that the version numbers are specified for the compilers. New versions with speed improvements are released frequently, so these timing data should not be taken to be prejudicial against more current or future versions produced by any vendor. The data serve mainly to show how extreme the speed differences can be.

Some of the gross differences are easy to interpret. Software-Toolworks $\mathrm{C}$ is unique in using fast singleprecision (4-byte) floating-point arithmetic, whereas most standard $\mathrm{C}$ compilers use double-precision (8-byte). However, the high accuracy of double-precision is not needed in these real-time applications. The data in the last row of Table 2 were obtained using an IBM AT with an INTEL 80287 arithmetic coprocessor. The speed of Microsoft and Turbo $\mathrm{C}$ with the combination of the AT

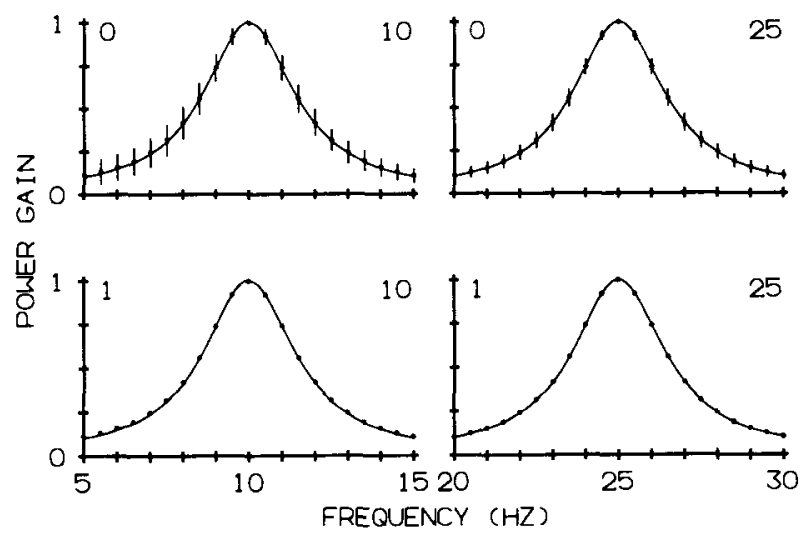

Figure 5. NEWSART power-windows showing the average power (dots) and the beat-oscillation magnitudes (vertical lines) for $m^{\prime}=0$ (top) and $m^{\prime}=1$ (bottom) for target frequencies off center at $10 \mathrm{~Hz}$ (left) and centered at $25 \mathrm{~Hz}$ in the range of estimable frequencies. For $m^{\prime}=1$, the vertical lines are not visible because they are shortor than the diameter of the dots. The other parameter values were $\Delta_{r}=.01$ sec, $m=1$, and $r=.9$. The smooth curves are the Student's $t$ densities from Equations 31-33.
Table 2

Estimated Durations (in msec) of the Minimal Achievable NEWSART Time-Step for Several Computers and C Compilers

\begin{tabular}{lcrrrrc}
\hline & \multicolumn{5}{c}{ Compiler } \\
\cline { 2 - 6 } \multicolumn{1}{c}{ Computer } & Toolworks & Microsoft & Turbo & Datalight & Mix \\
and Compiler & $3.2 \mathrm{M}$ & 4.00 & \multicolumn{1}{c}{1.5} & 3.01 & 2.2 .0 \\
\hline IBM-PC 8088 & 9.43 & 24.46 & 28.29 & 32.40 & 68.49 \\
Slicer 80188 & 4.78 & 13.64 & 15.02 & 17.01 & 38.79 \\
Slicer 80186 & 3.06 & 8.40 & 9.31 & 10.81 & 25.63 \\
IBM-AT 80286 & 2.59 & 5.83 & 6.43 & 8.10 & 19.30 \\
IBM-AT 80286/7 & 2.30 & .93 & .94 & 2.70 & 19.31 \\
\hline
\end{tabular}

80286 and 80287 is remarkable. Apparently, the other compilers (in the specified versions) do not fully exploit the speed of the 80287. Most differences among computers are predicted roughly from their specifications. The IBM-PC runs at $4.77 \mathrm{MHz}$, whereas the Slicer 80188 and 80186 run at $8 \mathrm{MHz}$. The 8088 and 80188 access memory one byte at a time, whereas the 80186 and 80286 transfer units of two bytes.

When $m$ and the number of target frequencies are increased, the approximate effect is to increase the times in Table 2 by a multiplicative factor equal to the product of $m$ and the number of target frequencies. An added NEWLIL stage with parameter $\boldsymbol{m}^{\prime}$ increases computation time, but not as much as would a corresponding increase in the NEWSART $m$ parameter value. Other design parameters have little effect on the computation times. As an example, consider the use of three target frequencies with $m=5$ and $m^{\prime}=0$. With Microsoft $\mathrm{C}$ on the AT with the 80287 , the minimal time-step would be about $14 \mathrm{msec}$ for a sampling rate of about $72 \mathrm{~Hz}$, giving a Nyquist frequency of about $36 \mathrm{~Hz}$.

\section{Programming a Real-Time Experiment}

The $\mathrm{C}$ language is a good choice for programming a real-time experiment. Indeed, the modules of Listings 1 and 2 can be used directly by such a program for most of the fundamental computations. The real-time program could look much like Test Program 1 of Listing 3. However, the real-time program would get data from an A/D converter rather than from a random-number generator. In addition, it would not contain the few lines in the test program that are intended for the speed measurements. Greater speed could be achieved in some computer systems by writing the real-time and speed-measurement programs so that integer arithmetic is used, rather than floating-point arithmetic. However, that technique requires extra programming to allow for scaling in order to avoid over- and underflow. The improvement might be small or large, depending on such things as whether an arithmetic coprocessor, such as the 80287 , is used. Indeed, the great variability of the timing data of Table 2 suggests that caution should be used in attempting to predict how much improvement there would be in any case.

The possible objectives and equipment resources are too varied for the useful formulation of a skeleton realtime program, so only these comments are offered, as- 
suming that the general outlines of the modules of Listings 1 and 2 are to be used.

The filpaka.c module of Listing 1 is designed to be truly modular because it embodies all the fundamental NEWSART and NEWLIL computations. If it is compiled by itself to be linked with an applications program, the only accessible global symbols are the names of the routines, $P_{-} Q_{-} R(), U_{-}$step(), and $h_{\text {_ }}$ step(). The module is thus quite immune to the inadvertant use of the same variable or function name for different purposes.

The setupa.c module of Listing 2 is not intended to be modular in the above sense. It is really no more than a part of the two calling programs, testla.c and test $2 a . c$, of Listings 3 and 4 . These calling programs overlap quite a bit in their needs for input, data storage, and initializing operations, so the setupa.c module is used to avoid having to rewrite the same code twice. It is intended to be included with any calling program that uses it, and the calling program must conform to the conventions for the names of the many common elements such as the define parameters and the global variables and arrays.

\section{CONCLUSION}

Adaptive digital estimation of a few spectral components is feasible in real time, and the capacities of readily available personal computers are adequate for applications such as feedback of EEG or automobile steering-wheel movement information. With continuing technological improvements in speed, the possible applications will extend to higher frequencies, to simultaneous estimations at more frequencies, and to greater choice among values of parameters that control the characteristics of the estimates.

A sliding time-window of the negative binomial form leads to recursive computations for economy of time, and to a spectral window of the form of Student's $t$ distribution. Knowledge of the form of this window is quite useful in evaluating proportionate power contributions from various frequency intervals.
A stage of NEWLIL filtering following the NEWSART filter can be added to increase the reliability of spectral estimates and to decrease the beat oscillations. The additional NEWLIL stage does not affect the widths or shapes of the average spectral windows determined by the NEWSART parameter values.

Soft copy of the listed $C$ programs will be supplied by the author on a 5.25-in. diskette in IBM MS-DOS $360 \mathrm{~K}$ format on request from scientific and educational users. Please include a blank diskette with request.

\section{REFERENCES}

Blackman, R. B., \& Tukey, J. W. (1958). The measurement of power spectra. New York: Dover.

BroOME, P. (1966). A frequency transformation for numerical filters. Proceedings of the IEEE, 52, 326-327.

EMERSON, P. L. (1983). Analysis of variance with Fourier analysis of coherent data. Behavior Research Methods \& Instrumentation, 15, 242-250.

EMERSON, P. L. (1985). FORTRAN subroutine for an EEG feedback digital filter. Behavior Research Methods, Instruments, \& Computers, 17, 469-473.

EMERSON, P. L. (1988). The best crude timer for MS-DOS implementations of C. Behavior Research Methods, Instruments, \& Computers, 20, 583-584.

FELLER, W. (1957). An introduction to probability theory and its applications: Vol. 1. New York: Wiley.

KAISER, J. F. (1966). Digital filters. In F. F. Kuo \& J. F. Kaiser (Eds.), System analysis by digital computer (pp. 218-277). New York: Wiley. Reprinted in Liu (1975).

KAPLAN, H. L. (1983). Correlations, contrasts, and components: Fourier analysis in a more familiar terminology. Behavior Research Methods \& Instrumentation, 15, 228-241.

Kendall, M. G., \& Stuart, A. S. (1963). The advanced theory of statistics: Vol. 1. Distribution theory (2nd ed.). New York: Hafner.

Liu, B. (Ed.) (1975). Digital filters and the fast Fourier transform. New York: Halstead.

Parzen, E. (1962). Stochastic processes. San Francisco: Holden-Day. STURGIS, S. P. (1983). A spectral-analysis tutorial with examples in FORTRAN. Behavior Research Methods \& Instrumentation, 15, 377-386.

Yost, M., JR., CoOPER, R. A., \& Bremner, F. J. (1983). Fourier analysis: A mathematical and geometric explanation. Behavior Research Methods \& Instrumentation, 15, 258-261.

\section{LISTING 1}

Fundamental NEWSART and NEWLIL Computations

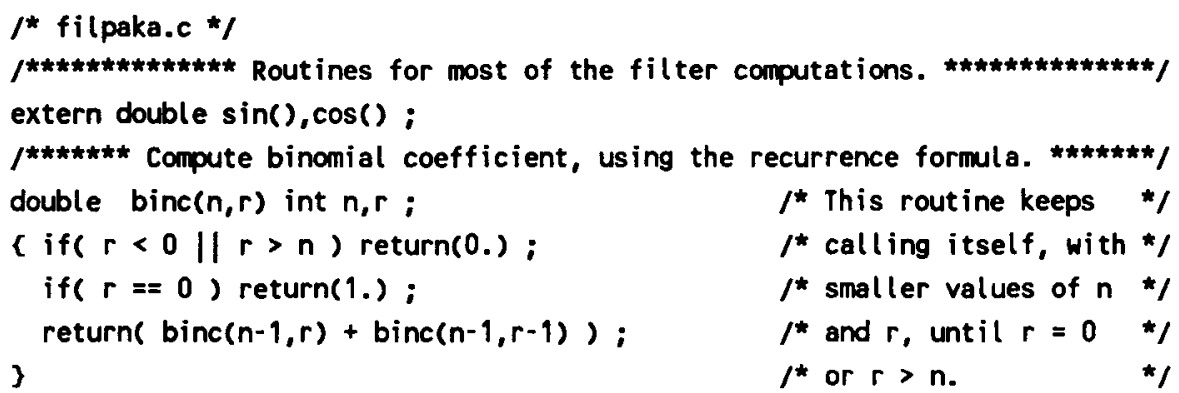




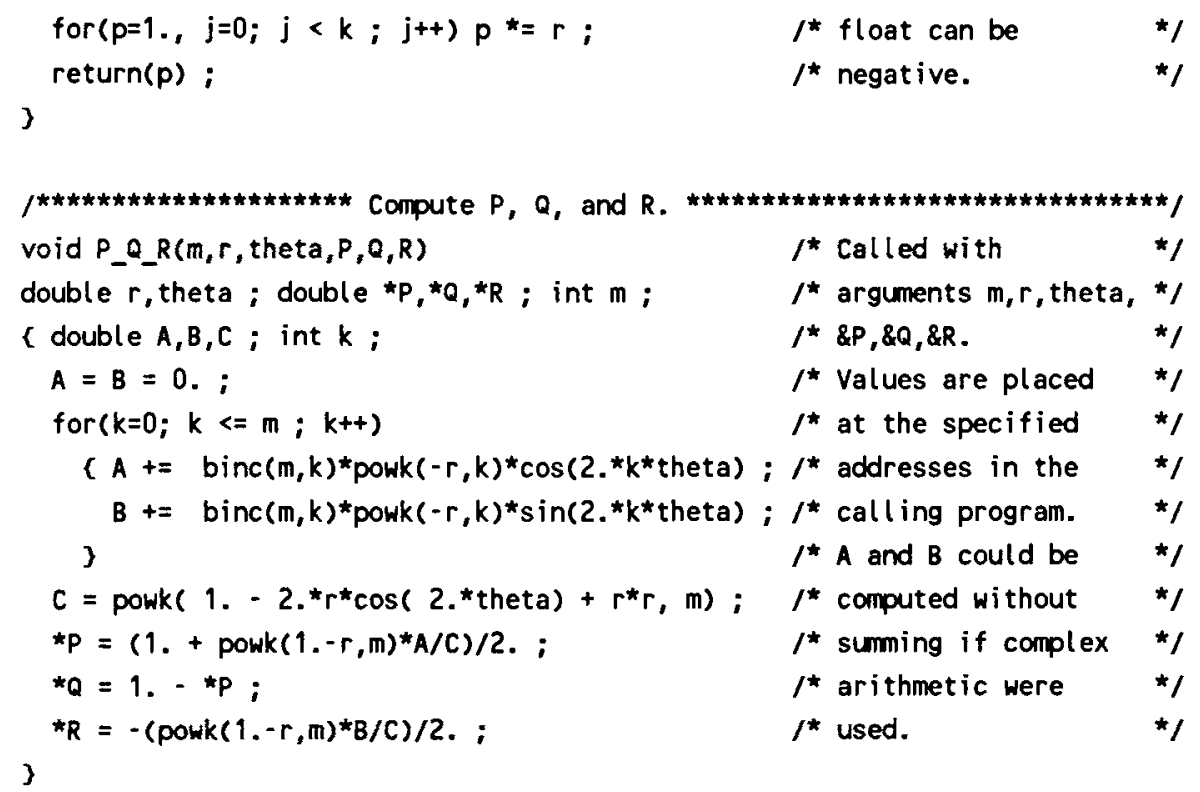


LISTING 2 (Continued)

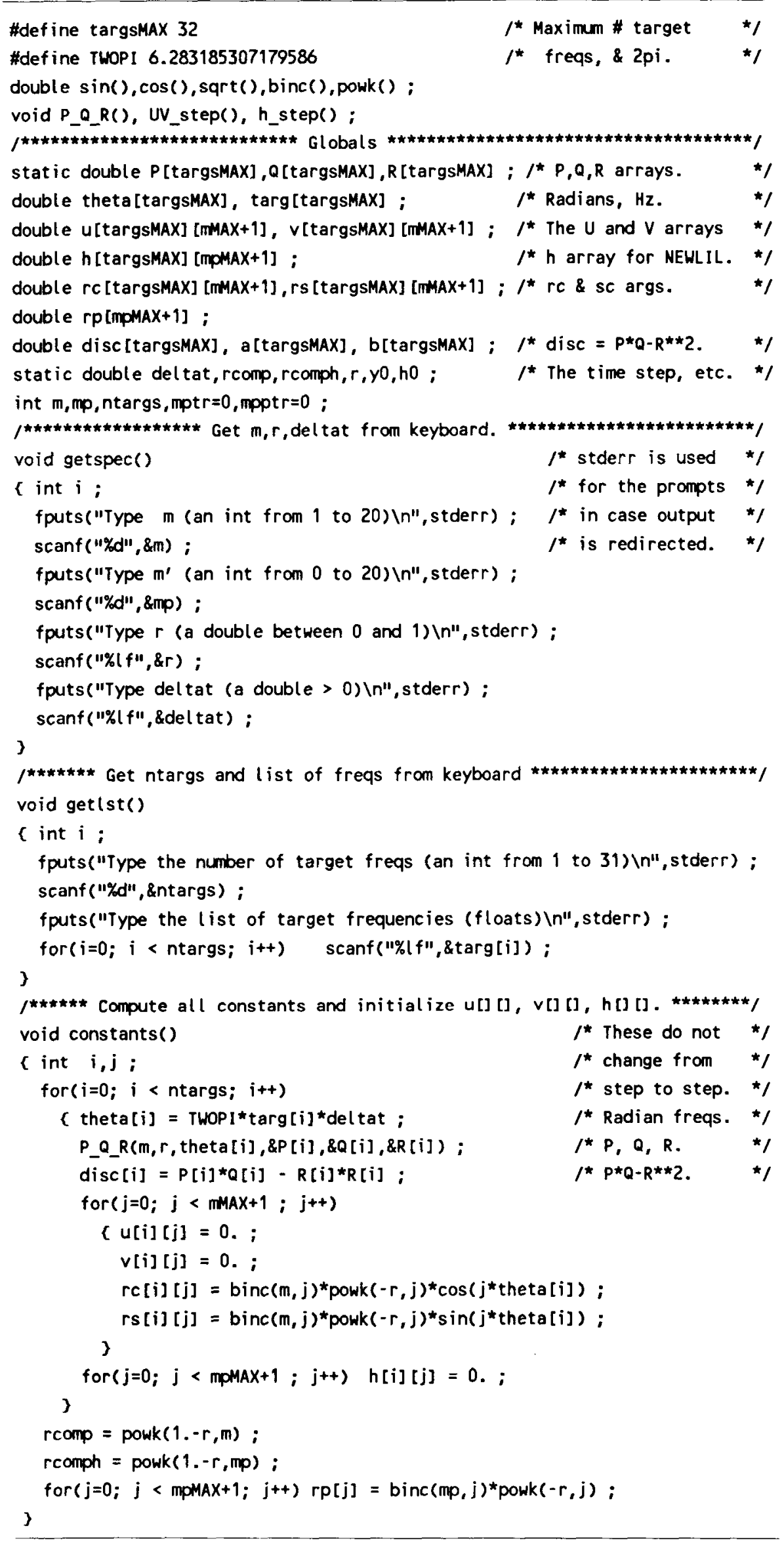


LISTING 3

Test Program 1, for Timing Tests

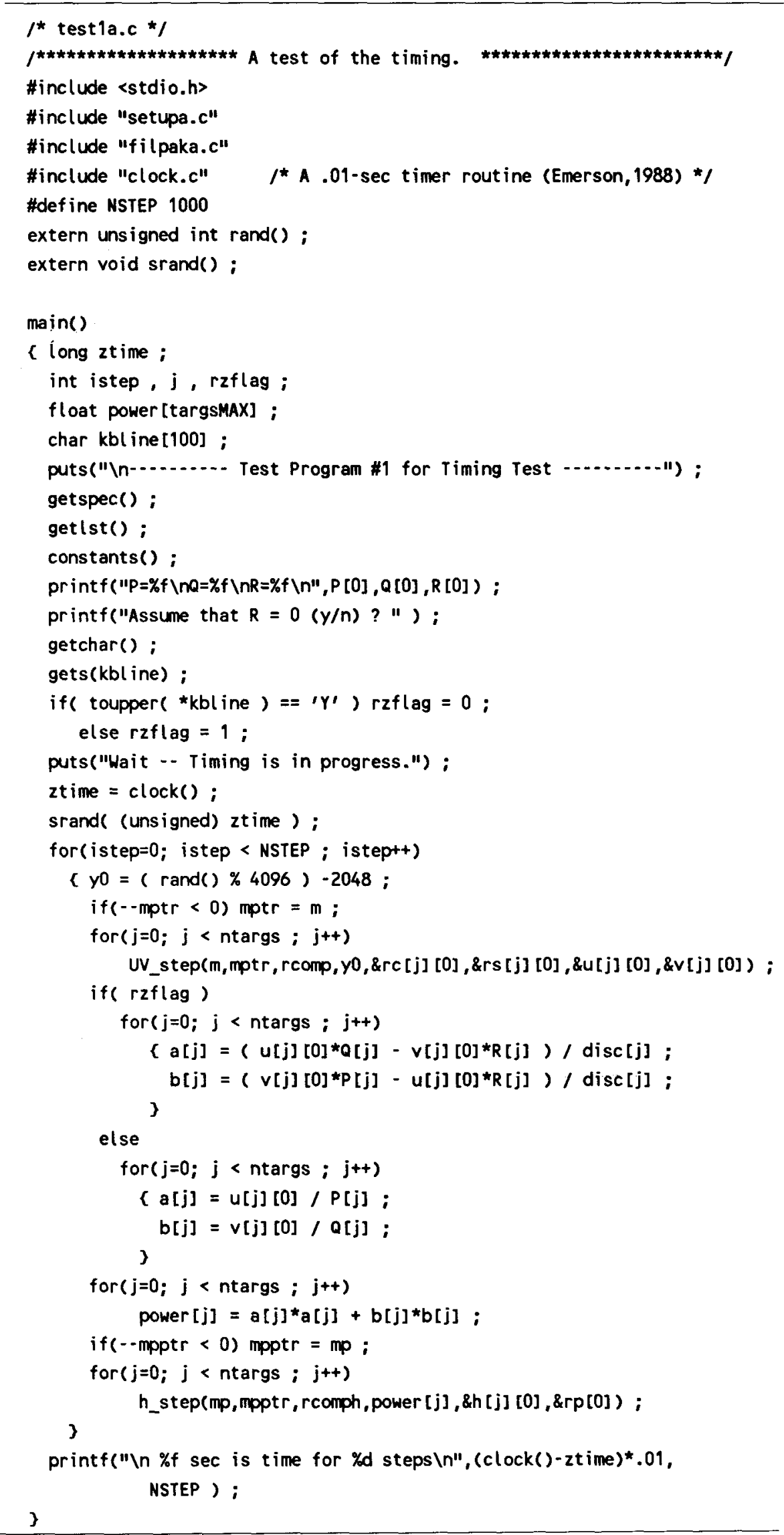


LISTING 4

Test Program 2, for Spectral-Window Tests

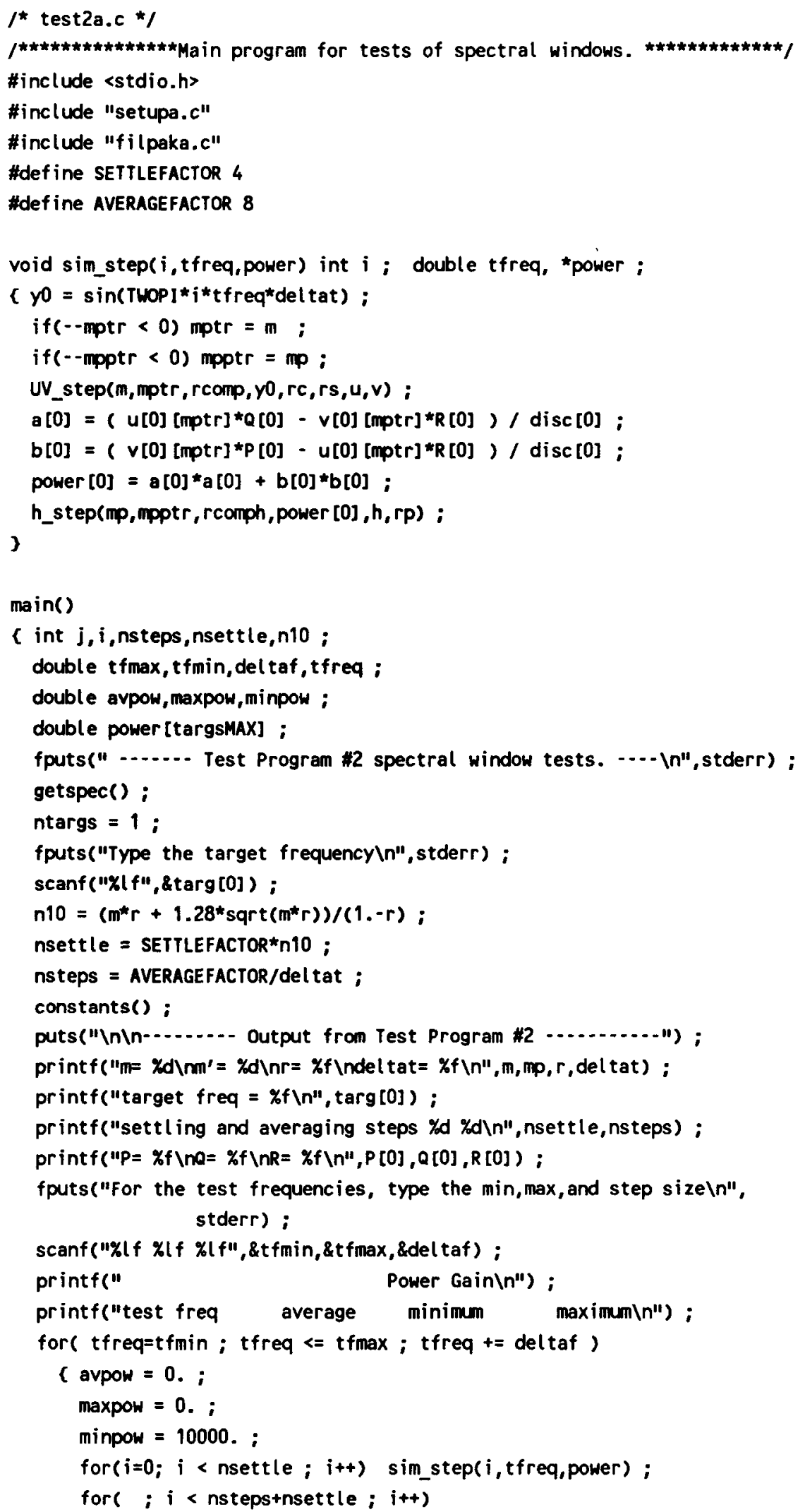




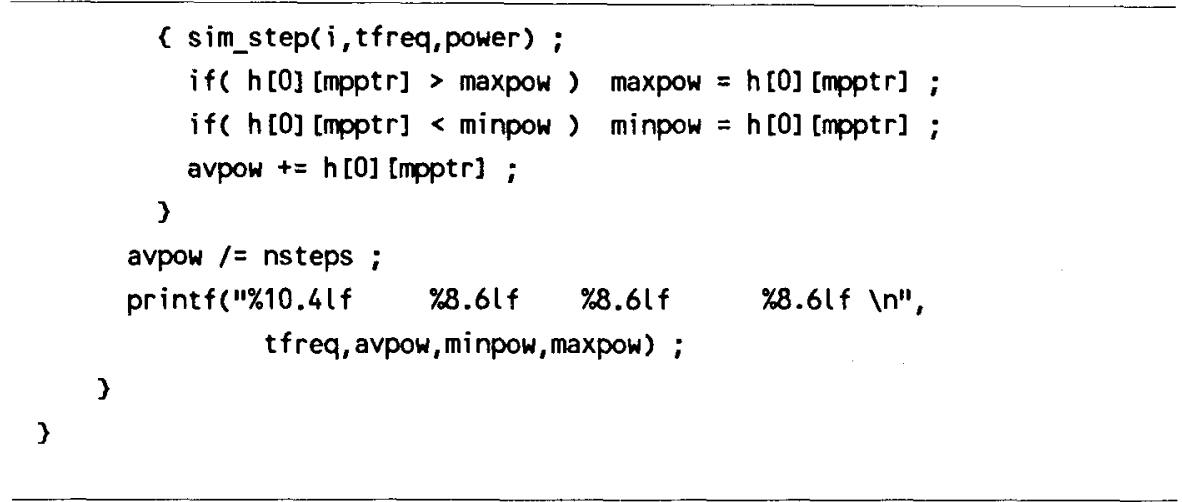

(Manuscript received July 21, 1988;

revision accepted for publication March 1, 1989.)

\title{
Notices and Announcements
}

\author{
19th Annual Meeting of the Society for Computers in Psychology \\ Atlanta, Georgia \\ November 16, 1989
}

The 19th Annual Meeting of the Society for Computers in Psychology will be held at the Hyatt Regency Hotel in Atlanta, Georgia on November 16, 1989. As usual, this meeting will feature papers on applications of computers to all areas of psychology-experimental, clinical, and educational.

For further information, contact Paula Goolkasian, Department of Psychology, University of North Carolina at Charlotte, Charlotte, NC 28223.

\section{Call for Papers}

Papers and symposia are sought for the 19th Annual Meeting of the Society for Computers in Psychology. The submission deadline is July 1, 1989. For more information, contact Darrell L. Butler, Department of Psychological Science, Ball State University, Muncie, IN 47306.

\section{Student Paper Competition}

The Society for Computers in Psychology will continue to sponsor an award for the outstanding student paper submitted for presentation at the annual meeting. Although the primary emphasis of the conference is on the use of computers in on-line, experimental applications, student papers in ary area of the application of computers to psychology are welcome. Papers may be theoretical, experimental, or applied in approach. Eligibility is open to (1) work done by a student currently enrolled in undergraduate or graduate courses, or (2) work done as part of a course, thesis, or other student research by a person who graduates in 1989. All papers submitted to the conference (including coauthored ones) in which the major contribution has been made by a student are eligible for the prize, and will be considered for presentation at the conference and subsequent publication in Behavior Research Methods, Instruments, \& Computers. The winning paper will be presented at the 1989 conference, and the author will receive a complimentary 1-year subscription to $\mathrm{Be}$ havior Research Methods, Instruments, \& Computers and a \$200 cash prize.

The submission deadline is July 1, 1989. Papers should be submitted in quadruplicate. A cover sheet should include the author's name, mailing address, telephone number, and academic affiliation, a 50-word abstract, and a note indicating that the paper is to be considered for the student award. Send these materials to Darrell L. Butler, Department of Psychological Science, Ball State University, Muncie, IN 47306. 